\title{
Constructing non-functionally expressible fuzzy implications
}

\author{
S. Massanet ${ }^{1}$ G. Mayor ${ }^{1}$ J. Torrens ${ }^{1}$ \\ ${ }^{1}$ University of the Balearic Islands \\ Carretera de Valldemossa, km 7.5, 07122 Palma, Spain
}

\begin{abstract}
This article discusses a new method for the construction of non-functionally expressible fuzzy implications. In a recent work, we have considered non-functionally expressible fuzzy implications from an implication function acting on the inputs followed by an aggregation process. Now, we first perform an aggregation of the inputs and then an implication function between the aggregated values. The main part is devoted to analyse under which conditions the two construction processes coincide.
\end{abstract}

Keywords: Fuzzy implications, implication functions, functionally expressible, morphisms, functional equation.

\section{Introduction}

One of the most important problems in fuzzy logic is the management of fuzzy conditionals of the type "If $p$, then $q$ " with $p$ and $q$ fuzzy statements. One method commonly used to do this management is through functions $I:[0,1] \times[0,1] \rightarrow[0,1]$ in such a way that, the truth value of the conditional is functionally stated from the truth values of the initial propositions $p$ and $q$. These functions $I$ are called (fuzzy) implication functions. Traditionally, it is accepted that any fuzzy concept must generalize the corresponding crisp concept and consequently, it is usually required to an implication function that $I(0,0)=I(0,1)=I(1,1)=1$ and $I(1,0)=0$; that is, its restriction to $\{0,1\}^{2}$ must coincide with the classical material implication, $p \rightarrow q \equiv \neg p \vee q$. Usually, antitonicity with the first variable and isotonicity with the second one are also required to implication functions leading to the most accepted definition $([2,6,10,12])$. Of course, several other conditions are also required to implication functions depending on the context, generally taken out from properties of the classical implication. This approach, known as the functionally expressible approach, is the most used in the literature and it has been extensively developed in last decades (see for instance the book [2] and the references therein).

Obviously the functionally expressible approach is only one of the possible methods. Moreover, in many cases fuzzy sets operations depend on the complete information about the involved fuzzy sets and thus, they need not be functionally expressible.
Alternatively, one can define fuzzy implications (as well as conjunctions, disjunctions, and other logical connectives) in an axiomatic way (see [11]), using the most accepted axioms for fuzzy implications. That is, decreasing in the first variable, increasing in the second one, and satisfying that the fuzzy implication must take crisp values (according to the rule $\neg p \vee q$ ) when the involved fuzzy sets take only crisp values. It is proved in [11] that not all fuzzy implications defined in this way are functionally expressible and many examples are done. In fact a method to construct non-functionally expressible fuzzy implications from two implications functions and an aggregation function having 0 and 1 as leftabsorbent elements is pointed out in the mentioned paper. Specifically, given a fuzzy set $A: X \rightarrow[0,1]$ on a universe $X$, and a fuzzy set $B: Y \rightarrow[0,1]$ on a universe $Y$, one can take the fuzzy implication

$(A \rightarrow B)(x, y)=F\left(I(A(x), B(y)), J\left(A\left(x_{0}\right), B\left(y_{0}\right)\right)\right)$,

where $I, J$ are implication functions, $F$ is an aggregation function with $F(0,1)=0$ and $F(1,0)=1$, and $x_{0}, y_{0}$ are fixed points in the corresponding universes. The previous construction corresponds to the idea of obtaining models of fuzzy implications such that the value $(A \rightarrow B)(x, y)$ depends not only on the values taken by $A$ and $B$ at the points $x$ and $y$ respectively (functionally expressible approach), but also on their values at other fixed points $x_{0}$ and $y_{0}$.

Keeping in mind the same idea we will present in this paper a new method to construct nonfunctionally expressible fuzzy implications using, similarly to the method proposed in Equation (1), two aggregation functions $F, G$ with 1 and 0 as left absorbing elements and an implication function $I$. Moreover, if we consider the same implication and aggregation functions in both approaches, we will investigate when they coincide, at least for some kinds of implication functions. In the case that the implication function $I$ satisfies the left neutrality principle, we will derive that this occurs if and only if the involved aggregation function $F$ depends only on the first variable, that is, when $F(x, y)=f(x)$ for all $x, y \in[0,1]$ being $f$ an increasing function with $f(0)=0$ and $f(1)=1$, that acts as a morphism of the implication function $I$. This of course leads to the study of morphisms of implication functions in general. 
The paper is organized as follows. In the next section we give some preliminaries that will be used along the paper. In Section 3 we deal with the new construction method previously mentioned, whereas in Section 4 we investigate when both methods (the one introduced here and the one studied in [11]) coincide, at least when the involved implication functions satisfy the left neutrality principle (recall that this is the case of the most used classes of implication functions), reducing such functional equation to the study of morphisms of implication functions. Then, the study of morphisms of implication functions $I$ when $I$ lies in the class of $(S, N)$-implications is managed in Section 5. In addition, some remarks on morphisms of other classes of implication functions are also pointed out. The paper ends with a conclusion section where some possible future work is also pointed out.

\section{Preliminaries}

In this section we recall some of the definitions and results that will be used along the paper. Any other basic facts not recalled here can be found in [9] for the case of t-norms, t-conorms and fuzzy negations, in [2] for implication functions, and in [3, 4, 8] for aggregation functions.

Definition 1 ([4]) A binary function $F:[0,1]^{2} \rightarrow$ $[0,1]$ is said to be an aggregation function if it is increasing in each variable and $F(0,0)=0$ and $F(1,1)=1$.

Given a non-empty set $X$, the collection of fuzzy subsets of $X$ can be represented by the set $[0,1]^{X}$ of functions from $X$ to the unit real interval $[0,1]$. In addition, given $k \in[0,1]$ we will denote by $k_{X}$ the fuzzy set $A$ such that $A(x)=k$ for all $x \in X$. Special examples of these constant fuzzy sets will be $0_{X}$ and $1_{X}$, the fuzzy sets $A$ and $B$ such that $A(x)=0$ and $B(x)=1$ for all $x \in X$, respectively.

Definition 2 (Definition 5 and Remark 1 in [11]) An operation $[0,1]^{X} \times[0,1]^{Y} \longrightarrow[0,1]^{X \times Y}$ : $(A, B) \longrightarrow A \rightarrow B$ is a fuzzy implication if the following conditions hold:

Im1) If $A \leq A^{\prime}$ then $A \rightarrow B \geq A^{\prime} \rightarrow B$ for all $B \in[0,1]^{Y}$, i.e., $\rightarrow$ is decreasing in the first variable.

Im2) If $B \leq B^{\prime}$ then $A \rightarrow B \leq A \rightarrow B^{\prime}$ for all $A \in[0,1]^{X}$, i.e., $\rightarrow$ is increasing in the second variable.

Im3) If $A \in\{0,1\}^{X}$ and $B \in\{0,1\}^{Y}$ then

$$
\begin{aligned}
& (A \rightarrow B)(x, y)= \begin{cases}0 & \text { if } A(x)=1 \text { and } B(y)=0, \\
1 & \text { otherwise. }\end{cases} \\
& \text { i.e., } \rightarrow \text { extends the crisp implication. }
\end{aligned}
$$

Definition 3 (Definition 1.15 in [6]) A binary operator $I:[0,1]^{2} \rightarrow[0,1]$ is said to be an implication function if it satisfies:
(I1) $I(x, z) \geq I(y, z)$ when $x \leq y$, for all $z \in$ $[0,1]$.

(I2) $I(x, y) \leq I(x, z)$ when $y \leq z$, for all $x \in$ $[0,1]$.

(I3) $I(0,0)=I(1,1)=1$ and $I(1,0)=0$.

Definition 4 (Definition 3 in [11]) We will say that an implication function $I:[0,1]^{2} \rightarrow[0,1]$ is trivial when it takes only the values 0 and 1 . That is, when

$$
I(x, y) \in\{0,1\} \quad \text { for all } \quad x, y \in[0,1] .
$$

Definition 5 (Definition 8 in [11]) A binary operation $F:[0,1]^{X} \times[0,1]^{Y} \longrightarrow[0,1]^{X \times Y}$ is said to be functionally expressible (f.e. for short) if there exists a function $f:[0,1] \times[0,1] \longrightarrow[0,1]$ such that for all $A \in[0,1]^{X}$ and $B \in[0,1]^{Y}$ it holds that $F(A, B)(x, y)=f(A(x), B(y))$ for all $(x, y) \in X \times Y$.

Proposition 1 (Proposition 5 in [11]) A fuzzy implication $\rightarrow$ is functionally expressible if, and only if, there exists an implication function $I$ : $[0,1] \times[0,1] \longrightarrow[0,1]$ such that $(A \rightarrow B)(x, y)=$ $I(A(x), B(y))$ for all $A \in[0,1]^{X}, B \in[0,1]^{Y}$, and $(x, y) \in X \times Y$.

Obviously not all fuzzy implications are functionally expressible and many examples were given in [11]. In particular, the following construction method was proved in [11].

Proposition 2 (Proposition 13 in [11]) Given two implication functions $I, J$, an aggregation function $F$ with $F(0,1)=0$ and $F(1,0)=1$, and fixed points $x_{0} \in X$ and $y_{0} \in Y$, then the function

$$
\rightarrow_{(F, I, J)}:[0,1]^{X} \times[0,1]^{Y} \rightarrow[0,1]^{X \times Y}
$$

defined by the expression $\left(A \rightarrow_{(F, I, J)} B\right)(x, y)=$ $F\left(I(A(x), B(y)), J\left(A\left(x_{0}\right), B\left(y_{0}\right)\right)\right)$ for all $A \in$ $[0,1]^{X}$ and $B \in[0,1]^{Y}$, is a fuzzy implication.

Just some minimal properties on the aggregation function $F$ and the implication function $I$ are needed to derive non-f.e. fuzzy implications through this method.

Proposition 3 (Proposition 14 in [11]) Let I, J be two implication functions and $F$ an aggregation function with $F(0,1)=0, F(1,0)=1$ and $F(x, 0)<F(x, 1)$ for all $x \in(0,1)$. Then the following statements are equivalent:

1. $\rightarrow_{(F, I, J)}$ is non-functionally expressible.

2. I is non-trivial.

We will give in the next section an alternative method to construct non-functionally expressible fuzzy implications from the same functions $F$ and implication functions $I, J$. 


\section{New construction method of non-f.e. fuzzy implications}

Note that with the method presented in Proposition 2 , the value of the constructed fuzzy implication at point $(x, y)$ is obtained by applying the aggregation function $F$ to the value of $I$ at point $(A(x), B(y))$ and the value of $J$ at point $\left(A\left(x_{0}\right), B\left(y_{0}\right)\right)$. A different but similar possibility consists on applying an aggregation function $F$ to the values $A(x), A\left(x_{0}\right)$, an aggregation function $G$ to the values $B(y), B\left(y_{0}\right)$, and finally an implication function $I$ to the aggregated values. Specifically,

Definition 6 Given two aggregation functions $F$ and $G$, an implication function $I$, and fixed points $x_{0} \in X$ and $y_{0} \in Y$, we define the function

$$
\rightarrow(I, F, G):[0,1]^{X} \times[0,1]^{Y} \rightarrow[0,1]^{X \times Y}
$$

by the expression

$$
\begin{aligned}
& \left(A \rightarrow_{(I, F, G)} B\right)(x, y)= \\
& I\left(F\left(A(x), A\left(x_{0}\right)\right), G\left(B(y), B\left(y_{0}\right)\right)\right)
\end{aligned}
$$

for all $A \in[0,1]^{X}$ and $B \in[0,1]^{Y}$.

In most of the cases it can be proved that the previous construction gives an implication function.

Proposition 4 Let $F, G$ be aggregation functions, $I$ an implication function, and $x_{0}, y_{0}$ arbitrary but fixed points of the corresponding universes $X, Y$, respectively. If $F, G$ satisfy $F(0,1)=G(0,1)=0$ and $F(1,0)=G(1,0)=1$, then the function

$$
\rightarrow(I, F, G):[0,1]^{X} \times[0,1]^{Y} \rightarrow[0,1]^{X \times Y}
$$

is always a fuzzy implication.

Proof Conditions $\operatorname{Im} 1$ ) and $\operatorname{Im} 2$ ) follow trivially from the increasingness of functions $F, G$ and the monotonicities of the implication function $I$. Similarly, condition $\operatorname{Im} 3$ ) follow from the behaviour of functions $F, G$ and $I$ at the values 0,1 .

Moreover, the operations constructed in this way are in general non-functionally expressible implication functions. To see this, let us recall that an implication function $I$ is said to satisfy the leftneutrality principle ${ }^{1}$, whenever

$$
I(1, y)=y \quad \text { for all } \quad y \in[0,1] .
$$

Then, the following result holds.

Proposition 5 Let $F, G$ be aggregation functions with $F(0,1)=G(0,1)=0$ and $F(1,0)=G(1,0)=$ 1. Let $I$ be an implication function and $x_{0}, y_{0}$ arbitrary but fixed points of the corresponding universes $X, Y$, respectively. Then the fuzzy implication $\rightarrow(I, F, G)$ is non-functionally expressible if one of the following conditions holds:

\footnotetext{
${ }^{1}$ Note that this is the case of most of the usual classes of implication functions, like $(S, N), R, Q L, D$ and Yager's implications.
}

1. There exists $a \in] 0,1[$ such that $F(a, 0)=0$ and $F(a, 1)=1$.

2. There exists $a \in] 0,1[$ such that $G(a, 0)=0$ and $G(a, 1)=1$.

3. I satisfies the left-neutrality principle and there are $a, b, b^{\prime} \in[0,1]$ such that $G(a, b) \neq G\left(a, b^{\prime}\right)$.

Proof Points 1 and 2 can be proved similarly, so let us detail only 1 . To do it, suppose that the fuzzy implication $\rightarrow_{(I, F, G)}$ is functionally expressible. Then there exists an implication function $J$ such that

$$
A \rightarrow_{(I, F, G)} B(x, y)=J(A(x), B(y))
$$

for all $A \in[0,1]^{X}, B \in[0,1]^{Y}$, and $x, y \in[0,1]$. Consider now $A, A^{\prime} \in[0,1]^{X}$ such that $A(x)=$ $A^{\prime}(x)=a, A\left(x_{0}\right)=0$ and $A^{\prime}\left(x_{0}\right)=1$, and $B, B^{\prime} \in[0,1]^{Y}$ such that $B(y)=B^{\prime}(y)=0$. Now, we have on one hand,

$$
\begin{aligned}
J(a, 0) & =J(A(x), B(y))=\left(A \rightarrow_{(I, F, G)} B\right)(x, y) \\
& =I\left(F(a, 0), G\left(0, B\left(y_{0}\right)\right)\right)=I(0,0)=1 .
\end{aligned}
$$

However, on the other hand, we obtain

$$
\begin{aligned}
J(a, 0) & =J\left(A^{\prime}(x), B^{\prime}(y)\right)=\left(A^{\prime} \rightarrow_{(I, F, G)} B^{\prime}\right)(x, y) \\
& =I\left(F(a, 1), G\left(0, B^{\prime}\left(y_{0}\right)\right)\right)=I(1,0)=0,
\end{aligned}
$$

obtaining a contradiction..

Let us now deal with the case when $I(1, y)=y$ and $G(a, b) \neq G\left(a, b^{\prime}\right)$. Suppose as above that the fuzzy implication $\rightarrow(I, F, G)$ is functionally expressible. Then there exists an implication function $J$ such that

$$
A \rightarrow_{(I, F, G)} B(x, y)=J(A(x), B(y))
$$

for all $A \in[0,1]^{X}, B \in[0,1]^{Y}$, and $x, y \in[0,1]$. Consider now $A, A^{\prime} \in[0,1]^{X}$ such that $A(x)=$ $A^{\prime}(x)=1$, and $B, B^{\prime} \in[0,1]^{Y}$ such that $B(y)=$ $B^{\prime}(y)=a, B\left(y_{0}\right)=b$, and $B^{\prime}\left(y_{0}\right)=b^{\prime}$. Now, we have on one hand,

$$
\begin{aligned}
J(1, a) & =J(A(x), B(y))=\left(A \rightarrow_{(I, F, G)} B\right)(x, y) \\
& =I\left(F\left(1, A\left(x_{0}\right)\right), G(a, b)\right)=I(1, G(a, b)) \\
& =G(a, b) .
\end{aligned}
$$

However, on the other hand, we obtain

$$
\begin{aligned}
J(1, a) & =J\left(A^{\prime}(x), B^{\prime}(y)\right)=\left(A^{\prime} \rightarrow_{(I, F, G)} B^{\prime}\right)(x, y) \\
& =I\left(F\left(1, A^{\prime}\left(x_{0}\right)\right), G\left(a, b^{\prime}\right)\right)=I\left(1, G\left(a, b^{\prime}\right)\right) \\
& =G\left(a, b^{\prime}\right) .
\end{aligned}
$$

and we get a contradiction since $G(a, b) \neq G\left(a, b^{\prime}\right)$.

Example 1 Let us consider the aggregation function $F$ given by

$$
F(a, b)= \begin{cases}1 & \text { if } a=1 \text { or }(a>0, b=1), \\ \min \{a, b\} & \text { otherwise. }\end{cases}
$$

Note that this aggregation function, which is obtained slightly modifying the minimum t-norm and is displayed in Figure 1, remains associative. 


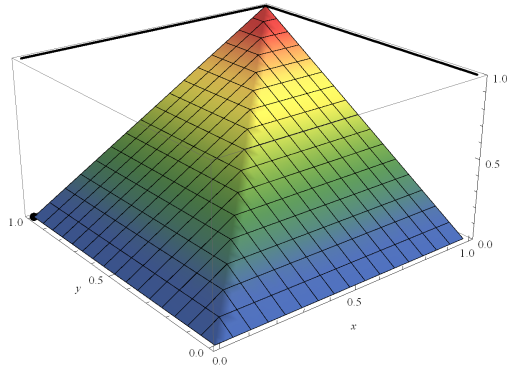

Figure 1: Aggregation function $F$ of Example 1.

Now, taking a non-trivial implication function I satisfying (NP), the corresponding fuzzy implication $\left(A \rightarrow_{(I, F, F)} B\right)(x, y)$

$$
=I\left(F\left(A(x), A\left(x_{0}\right)\right), F\left(B(y), B\left(y_{0}\right)\right)\right)
$$

for all $x, x_{0} \in X, y, y_{0} \in Y, A \in[0,1]^{X}$ and $B \in[0,1]^{Y}$ is not functionally expressible using the previous result. Let us illustrate how this operator is applied. If we consider the fuzzy sets $A \in[0,1]^{X}$ and $B \in[0,1]^{Y}$ and $x_{0} \in X, y_{0} \in Y$ with $0<A\left(x_{0}\right), B\left(y_{0}\right)<1$, which are displayed in Figure 2, we obtain the resulting values of Table 1.

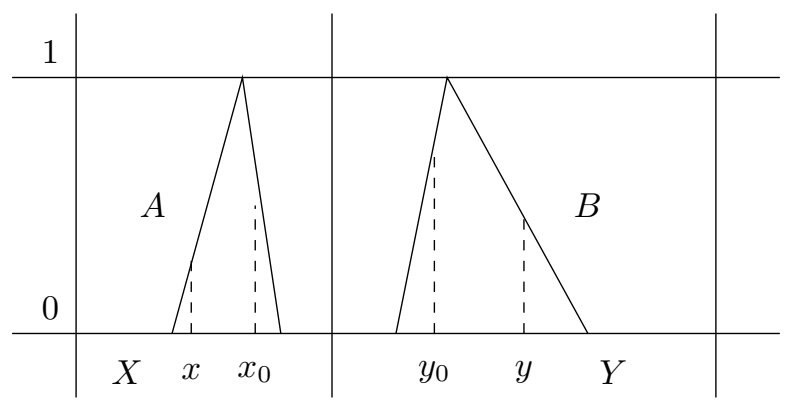

Figure 2: Fuzzy sets $A$ and $B$ of Example 1.

Now, if for instance, we consider the KleeneDienes implication $I_{K D}(x, y)=\max \{1-x, y\}$ for all $x, y \in[0,1]$, we obtain the results depicted in Table 2.

\section{When both construction methods coincide}

Comparing the method given in the section above $\left(\rightarrow_{(I, F, G)}\right)$ and the one given in $[11]\left(\rightarrow_{(F, I, J)}\right)$ one can wonder when both methods coincide if we consider the same aggregation and implication functions in both cases. That is, we want to investigate for which implication functions $I$, and aggregation functions $F$ with $F(0,1)=0$ and $F(1,0)=1$, it is satisfied

$$
\rightarrow_{(F, I, I)}=\rightarrow_{(I, F, F)}
$$

or equivalently, we want to solve the following functional equation

$$
F(I(x, y), I(z, t))=I(F(x, z), F(y, t))
$$

for all $x, y, z, t \in[0,1]$.

We will limit our study to implication functions satisfying the left-neutrality principle. In this case we have the following result.

Proposition 6 Let $F$ be an aggregation functions with $F(0,1)=0$ and $F(1,0)=1$, and let $I$ be an implication function satisfying the left-neutrality principle. Then the following items are equivalent:

1. F and I satisfy Equation (2).

2. There is an increasing function $f:[0,1] \rightarrow$ $[0,1]$ with $f(0)=0, f(1)=1$ and such that $F(x, y)=f(x)$ and

$$
f(I(x, y))=I(f(x), f(y))
$$

for all $x, y \in[0,1]$.

Proof First of all suppose that $F, I$ satisfy Equation (2). Note that taking $x=1$ and $z=0$ in this equation we have

$$
F(I(1, y), I(0, t))=I(F(1,0), F(y, t))
$$

and consequently $F(y, 1)=F(y, t)$ for all $y, t \in$ $[0,1]$, that is, $F$ does not depend on the second variable. So, taking $f:[0,1] \rightarrow[0,1]$ defined by $f(x)=F(x, y)$ for any $y \in[0,1]$, we obtain an increasing function with $f(0)=0$ and $f(1)=1$. Moreover, with this notation Equation (2) can be rewritten as

$$
f(I(x, y))=I(f(x), f(y)) .
$$

The converse follows by a simple computation.

In view of the previous proposition we will adopt the following definition.

Definition 7 Given an implication function $I$, an increasing function $f:[0,1] \rightarrow[0,1]$ with $f(0)=0$, $f(1)=1$ and such that

$$
f(I(x, y))=I(f(x), f(y))
$$

for all $x, y \in[0,1]$, will be called a morphism of the implication function I, or simply an I-morphism.

Thus, with this definition and Proposition 6 we have that the fuzzy implications $\rightarrow_{(F, I, I)}$ and $\rightarrow_{(I, F, F)}$ coincide if and only if the function $F$ depends only on the first variable and it is given through an increasing function $f$ which is an $I$ morphism. So, to conclude when both construction methods coincide, we only need to deal with morphisms of implication functions and we will do it in the next section.

\section{Morphisms of implication functions}

Let us begin this section with some technical results. First, recall that given an implication function $I$, its natural negation $N_{I}$ is defined by

$$
N_{I}(x)=I(x, 0) \text { for all } x \in[0,1] .
$$

The first result dealing with $I$-morphisms is related to the natural negation. 


\begin{tabular}{|c|c|c|c|}
\hline$A(x)$ & $B(y)$ & 1 & $(0,1)$ \\
\hline 0 & 1 & 1 & 1 \\
\hline 1 & 0 & 1 & $\min \left\{B(y), B\left(y_{0}\right)\right\}$ \\
\hline$(0,1)$ & $I\left(\min \left\{A(x), A\left(x_{0}\right)\right\}, 0\right)$ & 1 & $I\left(\min \left\{A(x), A\left(x_{0}\right)\right\}, \min \left\{B(y), B\left(y_{0}\right)\right\}\right)$ \\
\hline
\end{tabular}

Table 1: Values of the non-functionally expressible fuzzy implication $\left(A \rightarrow_{(I, F, F)} B\right)(x, y)$ of Example 1 .

\begin{tabular}{|c|c|c|c|}
\hline$A(x)$ & 0 & 1 & $(0,1)$ \\
\hline 0 & 1 & 1 & 1 \\
\hline 1 & 0 & 1 & $\min \left\{B(y), B\left(y_{0}\right)\right\}$ \\
\hline$(0,1)$ & $1-\min \left\{A(x), A\left(x_{0}\right)\right\}$ & 1 & $\max \left\{1-\min \left\{A(x), A\left(x_{0}\right)\right\}, \min \left\{B(y), B\left(y_{0}\right)\right\}\right\}$ \\
\hline
\end{tabular}

Table 2: Values of the non-functionally expressible fuzzy implication $\left(A \rightarrow_{(I, F, F)} B\right)(x, y)$ with $I=I_{K D}$ of Example 1.

Lemma 1 Let $I$ be an implication function and $f$ : $[0,1] \rightarrow[0,1]$ an $I$-morphism. Then $f$ commutes with the natural negation $N_{I}$.

Proof Just take $y=0$ in Equation (3).

We concentrate now our study in some usual classes of implications function, especially in $(S, N)$ implications. This class of implications is given from a t-conorm $S$ and a negation $N$ through the equality

$$
I_{S, N}(x, y)=S(N(x), y) \quad \text { for all } x, y \in[0,1] .
$$

Note that $(S, N)$-implications always satisfy the left neutrality principle. Moreover, in this case, the natural negation of $I_{S, N}$ is the proper negation $N^{2}$. Based on this fact, one can prove the following proposition in the general case.

Proposition 7 Let $I_{S, N}$ be an $(S, N)$-implication and $f:[0,1] \rightarrow[0,1]$ an increasing function with $f(0)=0$ and $f(1)=1$. Then the following items are equivalent.

i) $f$ is an $I_{S, N}$-morphism.

ii) $f$ commutes with $N$ and

$$
f(S(N(x), y))=S(f(N(x)), f(y)), \quad x, y \in[0,1]
$$

Proof First, let us prove that $i) \Rightarrow i i$ ). If $f$ is an $I_{S, N}$-morphism, then using Lemma $1, f$ commutes with $N_{I_{S, N}}=N$. In addition, we have

$$
\begin{aligned}
f\left(I_{S, N}(x, y)\right) & =I_{S, N}(f(x), f(y)) & \Rightarrow \\
f(S(N(x), y)) & =S(N(f(x)), f(y)) & \Rightarrow \\
f(S(N(x), y)) & =S(f(N(x)), f(y)) . &
\end{aligned}
$$

Reciprocally, the result follows just reversing the arguments above.

\footnotetext{
${ }^{2}$ For more details on this class of implication functions see [1] or $[2]$
}

In particular, Equation (4) becomes $f(S(x, y))=$ $S(f(x), f(y))$ for all $x$ in the range of $N$ and $y \in$ $[0,1]$. Therefore, the following corollary is immediate.

Corollary 1 Let $I_{S, N}$ be an $(S, N)$-implication with $N$ continuous and $f:[0,1] \rightarrow[0,1]$ an increasing function with $f(0)=0$ and $f(1)=1$. Then the following items are equivalent.

i) $f$ is an $I_{S, N}$-morphism.

ii) $f$ commutes with $N$ and $f$ is a morphism of the $t$-conorm $S$.

Thus, morphisms of $(S, N)$-implications with $N$ continuous are simply morphisms of the t-conorm $S$ that commute with the negation $N$. Morphisms of t-conorms in the framework of De Morgan triplets were already studied in [7]. However, note that in that paper the authors only dealt with isomorphisms $f$ with the assumptions that $f$ is strictly increasing and continuous. On the contrary, our study involves increasing functions in general, not necessarily continuous nor strictly increasing.

In what follows we will deal only with $(S, N)$ implications with $S$ a continuous t-conorm. We have the following results on morphisms of Archimedean t-conorms.

Proposition 8 Let $f:[0,1] \rightarrow[0,1]$ be an increasing function with $f(0)=0$ and $f(1)=1$ and $S$ an strict or a nilpotent t-conorm. Then $f$ is a morphism of the t-conorm $S$ if and only if $f$ is the identity function.

Consequently, we have the following corollary.

Corollary 2 Let $I_{S, N}$ be an $(S, N)$-implication with $S$ an strict or a nilpotent $t$-conorm, and $N$ a continuous negation. Then the only $I_{S, N}$-morphism is the identity function.

On the other hand, in the case of the maximum $\mathrm{t}$-conorm we have the following result. 
Proposition 9 Let $f:[0,1] \rightarrow[0,1]$ be a function with $f(0)=0$ and $f(1)=1$. Then $f$ is a morphism of the maximum t-conorm if and only if $f$ is increasing.

So, the following corollary holds.

Corollary 3 Let $N$ be a fuzzy negation and $f$ an increasing function with $f(0)=0$ and $f(1)=1$. Let $I$ be the $(S, N)$-implication given by $I_{N}(x, y)=$ $\max (N(x), y)$ for all $x, y \in[0,1]$. Then $f$ is an $I_{N^{-}}$ morphism if and only if $f$ commutes with $N$.

Thus, given a fuzzy negation $N, f$ is an $I$-morphism when $I$ is given by $I(x, y)=$ $\max (N(x), y)$ for a $x, y \in[0,1]$, if and only if $f$ commutes with $N$. Since continuous negations $N$ have a unique fixed point, it is clear that functions $f$ commuting with $N$ must satisfy $f(s)=s$, where $s$ is the fixed point of $N$. Moreover, when $N$ is strict we have the following proposition.

Proposition 10 Let $f:[0,1] \rightarrow[0,1]$ be an increasing function with $f(0)=0$ and $f(1)=1$, and $N$ a strict fuzzy negation with fixed point $s$. Then the following items are equivalent.

i) $f$ commutes with $N$.

ii) There is an increasing function $f_{0}:[0, s] \rightarrow$ $[0, s]$ with $f_{0}(0)=0, f_{0}(s)=s$ and such that $f$ is given by

$$
f(x)= \begin{cases}f_{0}(x) & \text { if } x \leq s \\ N\left(f_{0}\left(N^{-1}(x)\right)\right) & \text { if } x>s\end{cases}
$$

Note that when $N$ is not continuous, other morphisms appear as we show in the following examples.

Example 2 Let us consider the least $(S, N)$ implication $I_{D}$ given by

$$
I_{D}(x, y)= \begin{cases}1 & \text { if } x=0, \\ y & \text { if } x>0 .\end{cases}
$$

This $(S, N)$-implication is generated from the least fuzzy negation $N_{D_{1}}$ and any $t$-conorm $S$. Then it is easy to show that the I-morphisms of $I_{D}$ are functions $f:[0,1] \rightarrow[0,1]$ fulfilling $f(0)=0, f(1)=1$ and $f(x)>0$ for all $x>0$.

Example 3 Let us consider the greatest $(S, N)$ implication $I_{W B}$ given by

$$
I_{W B}(x, y)= \begin{cases}1 & \text { if } x<1 \\ y & \text { if } x=1 .\end{cases}
$$

This $(S, N)$-implication is generated from the greatest fuzzy negation $N_{D_{2}}$ and any t-conorm $S$. Then it is easy to show that the I-morphisms of $I_{W B}$ are functions $f:[0,1] \rightarrow[0,1]$ fulfilling $f(0)=0$, $f(1)=1$ and $f(x)<1$ for all $x<1$.
Other families of implication functions satisfying also the left neutrality principle are $R, Q L, D$ and Yager's implications. On the contrary to what happens with $(S, N)$-implications in the continuous Archimedean cases, for some of these other classes of implication functions there are other $I$-morphisms than the identity function.

For instance, consider the case of $R$-implications, which are given by

$I_{T}(x, y)=\sup \{z \in[0,1] \mid T(x, z) \leq y\}, \quad x, y \in[0,1]$

where $T$ is a t-norm. When $T$ is the minimum or the product t-norm, the corresponding $R$-implications have other morphisms than the identity function as the following examples show.

Example 4 Consider the Gödel implication $I_{G D}$, the residual implication from the minimum t-norm, which is given by

$$
I_{G D}(x, y)= \begin{cases}1 & \text { if } x \leq y \\ y & \text { if } x>y\end{cases}
$$

It is straightforward to prove that the strictly increasing functions $f:[0,1] \rightarrow[0,1]$ with $f(0)=0$ and $f(1)=1$ are the morphisms of $I_{G D}$.

Example 5 Take now the Goguen implication $I_{G G}$, the residual implication from the product $t$ norm, which is given by

$$
I_{G G}(x, y)= \begin{cases}1 & \text { if } x \leq y, \\ \frac{y}{x} & \text { if } x>y .\end{cases}
$$

The functions $f:[0,1] \rightarrow[0,1]$ such that $f(x)=x^{c}$ for some $c>0$ are morphisms of $I_{G G}$. However, these are not the only morphisms of this $R$ implication since the function $h_{0}:[0,1] \rightarrow[0,1]$ given by

$$
h_{0}(x)= \begin{cases}0 & \text { if } x=0 \\ 1 & \text { if } x>0 .\end{cases}
$$

is also a morphism of $I_{G G}$.

Now, let us briefly study the Yager's implications. There are two classes of Yager's implications, namely, $f$ - and $g$-generated implications. We will focus on the first one.

Definition 8 ([14], [2]) Let $f:[0,1] \rightarrow[0, \infty]$ be a strictly decreasing and continuous function with $f(1)=0$. The function $I:[0,1]^{2} \rightarrow[0,1]$ defined by

$$
I(x, y)=f^{-1}(x \cdot f(y)), \quad x, y \in[0,1]
$$

with the understanding $0 \cdot \infty=0$, is called an $f$ generated implication. The function $f$ is called an $f$-generator of the function I. In this case, we will write $I_{f}$ instead of I to emphasize the apparent relation. 
In this family, there are two subclasses depending on the fact that the corresponding generator $f$ takes a finite value at 0 or not, that is, if $f(0)<+\infty$ or $f(0)=+\infty$. It is known that in the first case the associate negation $N_{I}$ is strict (see Proposition 3.1.6 in [2]) and, in fact the corresponding $f$-generated implication is an $(S, N)$-implication with $S$ an strict t-conorm (see Theorem 6 in [13]). Thus, when $I_{f}$ is an $f$-generated implication with $f(0)<+\infty$ the only $I_{f}$-morphism is the identity function as it was proved in this section previously.

So, let us deal with the case of $f$-generated implications with $f(0)=+\infty$. In this case, the associated negation is always the Gödel negation $N_{\mathbf{D}_{\mathbf{1}}}$, that is,

$$
N_{I}(x)=N_{\mathbf{D}_{\mathbf{1}}}(x)= \begin{cases}1 & \text { if } x=0 \\ 0 & \text { otherwise }\end{cases}
$$

Thus, if $h$ is an $I_{f}$-morphism, the necessary condition that $h$ commutes with $N_{I_{f}}$ is always guaranteed by the fact that $f(0)=0$ and $f(1)=1$. Moreover, in this case there are other morphisms than the identity function. Take for instance the function $h_{0}$, previously defined in this section. Then it is an easy computation that $h_{0}$ is an $I_{f}$-morphism for any $f$-generated implication with $f(0)=+\infty$. However, the problem of determining all the morphisms of Yager's $f$-generated implications is one of our future goals.

Similarly, as a future work we want to deal with morphisms of $R$-implications and Yager's implications in general, as well as $Q L$ and $D$-implications.

\section{Acknowledgement}

This paper has been supported by the Spanish Grants MTM2009-10320 and MTM2009-10962, both with Feder support.

\section{References}

[1] M. Baczyński and B. Jayaram, (S,N)- and Rimplications: A state-of-the-art survey, Fuzzy Sets and Systems, Vol. 159:1836-1859, 2008.

[2] M. Baczyński and B. Jayaram, Fuzzy Implications, Studies in Fuzziness and Soft Computing, vol. 231, Springer, Berlin Heidelberg, 2008.

[3] G. Beliakov, A. Pradera, T. Calvo, Aggregation Functions: A Guide for Practicioners, in the series: Studies in Fuzziness and Soft Computing, 221, Springer, Berlin, Heidelberg, 2007.

[4] T. Calvo, G. Mayor and R. Mesiar, editors, Aggregation Operators. New trends and applications, in the series Studies in Fuzziness and Soft Computing, 97, Springer-Verlag, 2002.

[5] S.J. Chen, C.L. Hwang, Fuzzy Multiple Attribute Decision Making: Methods and Applications, Springer, Berlin, Heidelberg, 1992.

[6] J.C. Fodor and M. Roubens, Fuzzy Preference Modelling and Multicriteria decision Support, in the Series "Theory and Decision Library, serie D: System theory, Knowledge Engineering and Problem Solving", Kluwer Academic Publishers, Dordrecht, 1994.

[7] P. Garcia and L. Valverde, Isomorphisms between De Morgan tripets, Fuzzy Sets and Systems, 30:27-36, 1989.

[8] M. Grabisch, J.L. Marichal, R. Mesiar and E. Pap, Aggregation functions, in the series: Encyclopedia of Mathematics and its Applications, 127, Cambridge University Press, 2009.

[9] E. P. Klement, R. Mesiar, and E. Pap, Triangular norms. Kluwer, Dordrecht, 2000.

[10] G.J. Klir and B. Yuan, Fuzzy sets and fuzzy logic (Theory and applications). Prentice Hall, 1995.

[11] S. Massanet, G. Mayor, R. Mesiar and J. Torrens, On fuzzy implications: an axiomatic approach, accepted with minor revision in International Journal of Approximate Reasoning, 2013.

[12] M. Mas, M. Monserrat, J. Torrens and E. Trillas, A survey on Fuzzy Implications Functions, IEEE Transactions on fuzzy systems, 15:11071121, 2007.

[13] S. Massanet and J. Torrens, Intersection of Yager's implications with QL and Dimplications, International Journal of Approximate Reasoning, 53:467-479. 2012.

[14] R.R. Yager, On some new classes of implication operators and their role in approximate reasoning, Information Sciences, 167: 193-216, 2004. 